PROCEEDINGS OF THE

AMERICAN MATHEMATICAL SOCIETY

Volume 126, Number 6, June 1998, Pages 1873-1882

S 0002-9939(98)04361-5

\title{
ON THE MULTIPLE POINTS OF IMMERSIONS IN EUCLIDEAN SPACES
}

\author{
ANDRÁS SZÜCS
}

(Communicated by Thomas Goodwillie)

\begin{abstract}
Given a self-transverse immersion of a closed, oriented manifold in a euclidean space and a natural number $i$ we compute the oriented cobordism class of the manifold of $i$-tuple points.
\end{abstract}

\section{$\S 0$. INTRODUCTION}

Let $f: M^{n} \rightarrow R^{n+k}$ be a generic smooth immersion of a closed smooth manifold $M^{n}$ in the euclidean space $R^{n+k}$ and let $\Delta_{i}(f)$ denote the manifold of $i$-fold points in the image. The manifold $\Delta_{i}(f)$ is immersed in $R^{n+k}$ by a (non-generic) immersion $g_{i}$; the image of the non-multiple points of $g_{i}$ is the set of those points in $R^{n+k}$ which have exactly $i$ preimages.

The aim of this paper is to investigate the topological characteristics of these manifolds $\Delta_{i}(f)$. In particular we compute their signatures and more generally all their Pontrjagin numbers, show that in many cases they have even Euler characteristics, and investigate whether the cobordism class of the manifold $M^{n}$ determines their cobordism class or not. It turns out that when the multiplicity $i$ is odd, then the oriented cobordism class $\left[\Delta_{i}(f)\right]$ of $\Delta_{i}(f)$ is determined by the oriented cobordism class of $M^{n}$, while for $i$ even the oriented cobordism class $\left[\Delta_{i}(f)\right]$ is independent of the cobordism class of $M^{n}$.

\section{§1. Formulation of the Results}

The prototype of this kind of result is that of Banchoff:

Theorem (Banchoff). If $f: M^{2} \rightarrow R^{3}$ is a generic smooth immersion of a closed surface in $R^{3}$, then $\chi\left(M^{2}\right) \equiv t(f)$ mod 2 , where $t(f)$ denotes the number of triple points of $f$.

Eccles and Lannes gave a complete answer to the following question: For which dimensions $n$ does there exist an immersion of a closed $n$-manifold in $R^{n+1}$ with an odd number of $(n+1)$-fold points? (See [4], [5] and [7].)

Only a few papers have dealt with the higher-dimensional multiple points manifolds. (See [17], [6], [14], [15], [16].)

In [1] we generalized Banchoff's theorem as follows:

Received by the editors November 19, 1996.

1991 Mathematics Subject Classification. Primary 57R42.

(C)1998 American Mathematical Society 
Theorem. Let $f: M^{2 t} \rightarrow R^{2 t+1}$ be a generic codimension one immersion of the closed even-dimensional manifold $M^{2 t}$. Then $\sum_{i=1}^{2 t+1} \chi\left(\Delta_{i}(f)\right) \equiv 0 \bmod 2$.

The present paper arose from an attempt to generalize this result to codimension 2 immersions of oriented manifolds. In particular, we prove

Theorem 1. Let $f: M^{n} \rightarrow R^{n+2}$ be a generic immersion of an oriented closed manifold $M^{n}$ in $R^{n+2}$. If $n \not \equiv 2 \bmod 4$, then all of the Euler characteristics $\chi\left(\Delta_{i}(f)\right)$ are even.

By a result of Stong [12] an oriented closed manifold immersed in a euclidean space with codimension 2 is an unoriented boundary. Then by the Rohlin-Wall exact sequence $\left(\Omega_{n} \stackrel{2}{\rightarrow} \Omega_{n} \rightarrow \mathfrak{N}_{n}\right)$ its Pontrjagin numbers are even. Consequently the next theorem generalizes the previous one.

Theorem 2. Let $f: M^{n} \rightarrow R^{n+k}$ be a generic immersion of an oriented closed manifold $M^{n}$ in $R^{n+k}$, where $k$ is even, $n+k \neq 1 \bmod 4$, and suppose that all the Pontrjagin numbers of $M^{n}$ are even. Then all of the Euler characteristics $\chi\left(\Delta_{i}(f)\right)$ are even.

Remark 1. Any $n$-dimensional manifold can be immersed in $R^{2 n}$ with a single double point. This shows, that the condition $n+k \neq 0$ is necessary, but I do not know to what extent.

Returning to the case of codimension 2 immersions, Stong has shown the following theorem.

Theorem (Stong). Let $f: M^{n} \rightarrow R^{n+2}$ be a generic immersion of an oriented closed manifold $M^{n}$ in $R^{n+2}$. If $n$ is not divisible by 4 , then $M^{n}$ is an oriented boundary. If $n=4 t$, then there is an integer $j$ such that $\left\langle\bar{P}_{1}^{t},\left[M^{n}\right]\right\rangle=(2 t+1) ! \cdot j$ and any other normal Pontrjagin number of $M^{n}$ is zero.

In the next theorem we compute the Pontrjagin numbers of the manifolds of $i$-tuple points $\Delta_{i}(f)$ of such a codimension 2 immersion when the dimension of the manifold $\Delta_{i}(f)$ is divisible by four. Let $I=\left(i_{1}, \ldots, i_{r}\right)$ be a partition of $\frac{1}{4} \cdot(n-2 i+2)=\frac{1}{4} \cdot \operatorname{dim}\left(\Delta_{i}(f)\right)$. Let us denote by $\bar{P}_{I}\left[\Delta_{i}(f)\right]$ the corresponding normal Pontrjagin number. Further we denote by $\left(\begin{array}{l}i \\ I\end{array}\right)$ the product $\left(\begin{array}{c}i \\ i_{1}\end{array}\right) \ldots\left(\begin{array}{c}i \\ i_{r}\end{array}\right)$. The normal Euler class of the immersion $f$ will be denoted in this whole paper by $\bar{e}$.

Theorem 3. Let $f$ be the same as in Stong's theorem and let $\operatorname{dim}\left(\Delta_{i}(f)\right)=n-$ $2 i+2 \equiv 0 \bmod 4$. Then

$$
\begin{gathered}
\bar{P}_{I}\left[\Delta_{i}(f)\right]=\frac{(-1)^{i-1}}{i}\left(\begin{array}{c}
i \\
I
\end{array}\right)\left\langle\bar{e}^{n / 2},\left[M^{n}\right]\right\rangle \\
=\frac{(-1)^{i-1}}{i}\left(\begin{array}{c}
i \\
I
\end{array}\right) \cdot(n / 2+1) \cdot(\text { algebraic number of }(n / 2+1) \text {-tuple points of } f) .
\end{gathered}
$$

In particular, if $n=4 t$ and $\left\langle\bar{P}_{1}^{t},\left[M^{n}\right]\right\rangle=(2 t+1) ! \cdot j$ for $j \in Z$, then

$$
\bar{P}_{I}\left[\Delta_{i}(f)\right]=\left(\begin{array}{l}
i \\
I
\end{array}\right) \frac{(2 t+1) !}{i} j \text { for any odd } i .
$$

If $n-2 i+2 \not \equiv 0 \bmod 4$, then both sides of all these equalities vanish trivially.

Example. Let us consider an immersion $f: M^{8} \rightarrow R^{10}$. By Stong's theorem there is an integer $j$ such that the cobordism class of $M^{8}$ in the group $\Omega_{8}=Z \oplus Z$ generated by $\left[C P^{4}\right]$ and $\left[C P^{2} \times C P^{2}\right]$ is equal to $\left[M^{8}\right]=12 j\left[C P^{4}\right]+20 j\left[C P^{2} \times C P^{2}\right]$. 
By our theorem the cobordism class of the triple points manifold $\Delta_{3}(f)$ which belongs to the group $\Omega_{4}=Z=\left\{\left[C P^{2}\right]\right\}$ is equal to $40 j\left[C P^{2}\right]$, and the algebraic number of 5 -tuple points is equal to $24 j$.

(The oriented cobordism classes of the double and quadruple points manifolds belong to the trivial groups $\Omega_{6}$ and $\Omega_{2}$.)

A final group of results again concerns immersions in arbitrary codimension $k$. First some more notation. Let us denote by $\tilde{\Delta}_{i}(f)=\left\{\left(x_{1}, \ldots, x_{i}\right) \mid \forall x_{j} \in\right.$ $M^{n}, f\left(x_{1}\right)=\ldots=f\left(x_{i}\right)$ and $x_{1}, \ldots, x_{i}$ are all different $\}$. Then $\tilde{\Delta}_{i}(f) \subset M \times \ldots \times$ $M$ ( $i$ factors) is invariant under the action of the symmetric group $S(i)$, this action restricted to $\tilde{\Delta}_{i}(f)$ is free, and the quotient $\tilde{\Delta}_{i}(f) / S(i)$ is $\Delta_{i}(f)$.

Remark 2. In computing the signature $\sigma\left(\Delta_{i}(f)\right)$ the only interesting case is when both $n$ and $k$ are even. Indeed, if $n$ is odd and $k$ is even then all the manifolds $\Delta_{i}(f)$ have odd dimensions. If $k$ is odd then the $S(i)$ action contains orientation reversing involutions on $\tilde{\Delta}_{i}(f)$, and so either the quotient $\Delta_{i}(f)$ is non-orientable or its components have no preferred orientations.

Therefore we shall suppose from now on that $n$ and $k$ are even. Denote by $L^{i}(M)$ the $i$-th power of Hirzebruch's $L$ class of the manifold $M$.

Theorem 4. Let $f: M^{n} \rightarrow R^{n+k}$ be a generic immersion ( $n$ and $k$ are even). Then for any $i$ the signature of the manifold $\Delta_{i}(f)$ is the following:

$$
\sigma\left(\Delta_{i}(f)\right)=\frac{1}{i}\left\langle\bar{e}^{(i-1)} L^{i}(M),[M]\right\rangle .
$$

Actually a similar formula holds for any Pontrjagin number of the multiple points manifolds. Let $P(M)$ denote the total Pontrjagin class of $M$. Let $P^{i}(M)$ be the $i$-th power of this class and let $q_{j}$ be its $j$-dimensional part. For any partition $J=\left(j_{1}, \ldots, j_{r}\right)$ denote by $P^{i}(M)_{J}$ the product $q_{j_{1}} \ldots q_{j_{r}}$ and by $P_{J}\left(\Delta_{i}(f)\right)$ the usual product $P_{j_{1}} \ldots P_{j_{r}}\left(\Delta_{i}(f)\right)$.

Theorem 5. $\left\langle P_{J}\left(\Delta_{i}(f)\right),\left[\Delta_{i}(f)\right]\right\rangle=\frac{(-1)^{i-1}}{i} \cdot\left\langle\bar{e}^{i-1} P^{i}(M)_{J},[M]\right\rangle$.

Corollary. a) All the Pontrjagin numbers of $\Delta_{i}(f)$ vanish if those of $M$ vanish and $i$ is odd, or if the normal Euler class $\bar{e}$ has finite order.

b) For any $i$ the oriented cobordism classes $\Delta_{2 i+1}(f)$ of the $2 i+1$-tuple points are completely determined (modulo a possible second order torsion element) by the oriented cobordism class of $M^{n}$.

Remark 3. Notice that the situation is just the opposite for the double points manifold $\Delta_{2}(f)$, as the theorem below (proved in [15]) shows. Let us denote by $\mathrm{Imm}^{S O}(n, k)$ the cobordism group of immersions of oriented $n$ dimensional manifolds in $R^{n+k}$.

Theorem. If $k$ is even and $n+1<2 k$, then modulo finite 2-primary groups the following isomorphism holds:

$$
\operatorname{Imm}^{S O}(n, k) \approx \Omega_{n} \oplus \Omega_{n-k}
$$

This isomorphism associates to the cobordism class $[f]$ of an immersion $f: M^{n} \rightarrow$ $R^{n+k}$ the pair $\left(\left[M^{n}\right],\left[\Delta_{2}(f)\right]\right)$.

This theorem shows that the cobordism classes $\left[M^{n}\right]$ and $\left[\Delta_{2}(f)\right]$ are completely independent. A similar result holds also for $n<3 k$ modulo finite 3 and 2 primary 
groups, see [16]. (In the range $n<3 k$ the cobordism class of $M^{n}$ cannot be of course any cobordism class, since $\bar{P}_{j}(M)=0$ if $j>k / 2$.)

Remark 4. The possible unoriented cobordism classes of the double point sets of immersions in euclidean space have been computed by Eccles in [6] for the special case when the domain is the $n$-sphere, and by the author in [14] when the double point sets are 2-dimensional but the domain manifolds can be arbitrary (possibly nonorientable) manifolds.

\section{§2. The homology Class ReALized By $\tilde{\Delta}_{i}(f)$ IN $M \times \ldots \times M$}

The key to the proofs of all the theorems listed above is the Main Lemma below.

Let us denote by $(M)^{i}$ the product $M \times \ldots \times M$ (i factors). Further denote by $\Delta_{i}(M)$ the (narrow) diagonal in $(M)^{i}$, i.e. $\Delta_{i}(M)=\{(x, \ldots, x) \mid x \in M\}$. The cohomology class dual to the homology class realized by $\Delta_{i}(M)$ in $(M)^{i}$ will be denoted by $\delta_{i}$. Let us denote by $b_{i}$ the cohomology class of $(M)^{i}$ dual to the homology class realized by the submanifold $\tilde{\Delta}_{i}(f)$. For $i=1$ we put $b_{1}=\delta_{1}=1$.

Main Lemma. (Remember that $n$ and $k$ are even.)

$$
b_{i}=(-1)^{i-1}(i-1) ! \cdot\left(\bar{e}^{i-1} \times 1 \times 1 \times \ldots \times 1\right) \cup \delta_{i} .
$$

Remark 5. Computations of the homology class realized by the projection $\pi$ of $\tilde{\Delta}_{i}(f)$ in $M$ may be found in [8], [9], [13]. (Although the first of these is known to be incorrect we have borrowed many ideas from it.) Herbert's formula expressing this homology class in $H_{*}(M)$ can be obtained in the special case when the target is a euclidean space from our Main Lemma by applying the slant product with the fundamental class of $(M)^{i-1}$ to both sides and noticing that the map $\pi: \tilde{\Delta}_{i}(f) \rightarrow$ $\pi\left(\tilde{\Delta}_{i}(f)\right)$ has degree $(i-1)$ !.

Proof of the Main Lemma. Let us denote by $l(s)$ the integer $(-1)^{s-1}(s-1)$ ! for any natural number $s$ strictly less than $i$. The product $\left(\bar{e}^{(s-1)} \times 1 \times \cdots \times 1\right) \cup \delta_{s}$ will be denoted by $\theta_{s}$ for any $s$ less than or equal to $i$. Now the proof goes by induction; suppose that for any $s<i$ we have $b_{s}=l(s) \cdot \theta_{s}$.

a) First we shall show that there exists an integer $l(i)$ such that $b_{i}=l(i) \cdot \theta_{i}$.

b) Then we show that $l(i)=(-1)^{i-1}(i-1)$ !.

We denote by $\mathcal{A}(i)$ the set of all set-theoretical partitions of the set $\{1,2, \ldots, i\}$.

For any $\alpha \in \mathcal{A}(i)$ denote by $M_{\alpha}$ the following subset of $(M)^{i}$ :

$$
M_{\alpha}=\left\{\left(x_{1}, \ldots, x_{i}\right) \mid x_{j_{1}}=x_{j_{2}} \text { iff } j_{1} \text { and } j_{2} \text { are in the same group of } \alpha\right\} .
$$

We define a partial ordering on $\mathcal{A}(i)$ as follows: $\alpha<\beta$ if $\beta$ is a refinement of $\alpha$, i.e. $\beta$ is obtained from $\alpha$ by dividing some of the groups into smaller groups. Let $\alpha_{0}$ be the smallest partition (when there is a single group containing all the numbers $1, \ldots, i)$. The greatest element of $\mathcal{A}(i)$ (when each group contains only one number) will be denoted by $\alpha_{t o p}$.

Notice that $M_{\alpha_{0}}$ is the narrow diagonal $\Delta_{i}(M)$, and $M_{\alpha_{\text {top }}}$ is the complement in $(M)^{i}$ of the fat diagonal:

$$
M_{\alpha_{\text {top }}}=\left\{\left(x_{1}, \ldots, x_{i}\right) \mid x_{j} \in M \text { and they are all different }\right\} .
$$

Let $\tilde{F}_{i}$ be the $i$ times product of $f$, i.e. $\tilde{F}_{i}=f \times \cdots \times f: M \times \cdots \times M \rightarrow R^{n+k} \times$ $\cdots \times R^{n+k}$ ( $i$ factors). Let us denote by $\tilde{B}_{\alpha}$ the set $\tilde{F}_{i}^{-1}\left(\Delta_{i}\left(R^{n+k}\right)\right) \cap M_{\alpha}$ and let 
$V_{\alpha} \subset \bar{V}_{\alpha} \subset U_{\alpha}$ be neighbourhoods of $\tilde{B}_{\alpha}$ such that $U_{\alpha} \cap U_{\alpha^{\prime}}$ if $\alpha \neq \alpha^{\prime}$. Such disjoint neighbourhoods exist because $f$-being an immersion - is a local embedding.

Let $F_{i}$ be a map close to $\tilde{F}_{i}$ and transverse to the narrow diagonal $\Delta_{i}\left(R^{n+k}\right)$ and coinciding with $\tilde{F}_{i}$ on $U_{\alpha} \backslash \bar{V}_{\alpha}$. Denote by $B_{\alpha}$ the submanifold $F_{i}^{-1}\left(\Delta_{i}\left(R^{n+k}\right)\right) \cap U_{\alpha}$ in $(M)^{i}$, and let $b_{\alpha}$ be the cohomology class dual to the homology class realized by $B_{\alpha}$.

Our task is to compute the class $b_{\alpha_{t o p}}$, which is the same as $b_{i}$. We shall do this using the following claims, which will be proved later.

Claim 1.

$$
\sum_{\alpha \in \mathcal{A}(i)} b_{\alpha}=0
$$

Claim 2.

$$
b_{\alpha_{0}}=\left(\bar{e}^{i-1} \times 1 \times \cdots \times 1\right) \cup \delta_{i} .
$$

Let us consider an $\alpha \in \mathcal{A}(i)$ different from the greatest element $\alpha_{\text {top }}$. Let $\alpha$ consist of $s$ groups $I_{1}, \ldots, I_{s}$ of cardinalities $i_{1}, \ldots, i_{s}$ respectively. Since $\alpha \neq \alpha_{\text {top }}$ one has $s<i$. Let us consider now the set $\mathcal{A}\left(I_{1}\right)$ of the partitions of the set $I_{1}$ with the partial ordering similar to that on $\mathcal{A}(i)$. Let $\alpha_{0}\left(I_{1}\right)$ be the smallest element in $\mathcal{A}\left(I_{1}\right)$. Define the submanifold $B_{\alpha_{0}\left(i_{1}\right)}$ in $(M)^{i_{1}}$ analogously to $B_{\alpha_{0}}$ in $(M)^{i}$. Let $\pi_{I_{1}}:(M)^{i} \rightarrow(M)^{i_{1}}$ be the obvious projection keeping only the coordinates with indices from $I_{1}$. Let $A_{1}(\alpha)$ be $\pi_{I_{1}}^{-1}\left(B_{\alpha_{0}\left(i_{1}\right)}\right)$. Let us define similarly the submanifolds $A_{2}(\alpha), \ldots, A_{s}(\alpha)$. Finally let us define $C_{\alpha}$ as follows. Pick one index from each of the groups $I_{1}, \ldots, I_{s}$, denote this set of indices by $S$, and consider the corresponding projection $\pi_{S}:(M)^{i} \rightarrow(M)^{s}$. Consider the element $\alpha_{t o p}(s)$, i.e. the greatest element in $\mathcal{A}(S)$, and the corresponding set $B_{\alpha_{t o p}(s)}$ in $(M)^{s}$. Put $C_{\alpha}=\pi_{S}^{-1}\left(B_{\alpha_{\text {top }}(s)}\right)$.

Claim 3.

$$
B_{\alpha}=\left(\bigcap_{j=1}^{s} A_{j}(\alpha)\right) \cap C_{\alpha}
$$

Let us denote by $a_{j}$ the cohomology class in $(M)^{i}$ dual to the homology class realized by $A_{j}(\alpha)$. Then $a_{j}=\pi_{I_{j}}^{*}\left(b_{\alpha_{0}\left(i_{j}\right)}\right)$. This cohomology class can be computed using Claim 2. For example, if $I_{j}=\left\{1, \ldots, i_{j}\right\}$, then

$$
a_{j}=\left(\bar{e}^{i_{j}-1} \times 1 \times \cdots \times 1\right) \cup \pi_{I_{j}}^{*}\left(\delta_{i_{j}}\right) .
$$

In general, in this product digits 1 appear in the places which do not belong to $I_{j}$.

The cohomology class dual to the class realized by $C_{\alpha}$ will be denoted by $c_{\alpha}$. This class can be computed as follows: $c_{\alpha}=\pi_{S}^{*}\left(b_{\alpha_{t o p}(s)}\right)$. Since $s<i$, by the hypothesis of the induction one has $b_{\alpha_{\text {top }}(s)}=l(s) \cdot\left(\theta_{s}\right)$. Therefore

$$
c_{\alpha}=l(s) \cdot \pi_{S}^{*}\left(\theta_{s}\right) .
$$

Now we compute the class $b_{\alpha}$. (Remember that $\alpha$ is any partition from $\mathcal{A}(i)$ different from the greatest one.) By Claim 3 one has $b_{\alpha}=\left(\prod_{j=1}^{s} a_{j}\right) \cup c_{\alpha}$.

Proposition. $b_{\alpha}=l(s) \cdot \theta_{i}$, where $s$ is the number of groups in the partition $\alpha$.

This proposition follows immediately from the previous description of $a_{j}, c_{\alpha}$ and $b_{\alpha}$ using the following "symmetrizing property" of the diagonal class $\delta_{i}$. 
Symmetrizing property. Let $u_{1}, \ldots, u_{i}$ be arbitrary cohomology classes in the ring $H^{*}\left(M^{n}\right)$. Then

$$
\left(u_{1} \times \cdots \times u_{i}\right) \cup \delta_{i}=\left(\left(u_{1} \cup \cdots \cup u_{i}\right) \times 1 \times \cdots \times 1\right) \cup \delta_{i} .
$$

Proof. For $i=2$ this follows easily from lemma 11.8 of [11], saying that $(a \times 1) \cup \delta_{2}=$ $(1 \times a) \cup \delta_{2}$. Indeed,

$$
\begin{aligned}
\left(\left(u_{1} \cup u_{2}\right) \times 1\right) \cup \delta_{2} & =\left(u_{1} \times 1\right) \cup\left(u_{2} \times 1\right) \cup \delta_{2} \\
& \left.=\left(u_{1} \times 1\right) \cup\left(1 \times u_{2}\right) \cup \delta_{2}=\left(u_{1} \times u_{2}\right) \cup \delta_{2} .\right)
\end{aligned}
$$

To prove it for arbitrary $i$ we use the fact that

$$
\delta_{i}=\left(\delta_{2} \times 1 \times \cdots \times 1\right) \cup\left(1 \times \delta_{2} \times 1 \times \cdots \times 1\right) \cup \cdots \cup\left(1 \times 1 \times \cdots \times 1 \times \delta_{2}\right) .
$$

Therefore we have computed all the classes in the sum $\sum b_{\alpha}=0$ except the one corresponding to $\alpha_{t o p}$. Since we have got $b_{\alpha}=l(s) \cdot \theta_{i}$ where $s$ is the number of groups in the partition $\alpha$ for any $\alpha \neq \alpha_{\text {top }}$, we see that $b_{\alpha_{\text {top }}}=l(i) \cdot \theta_{i}$ for an integer $l(i)$. In order to prove that $l(i)=(-1)^{i-1}(i-1)$ ! notice that by Claim 3 the integers $l(j)$ satisfy the equality $\sum_{\alpha \in \mathcal{A}(i)} l(s)=0$. Therefore if $d(s, i)$ denotes the number of those $\alpha \in \mathcal{A}(i)$ which consist of $s$ groups, then we have $\sum_{s=1}^{s=i} d(s, i) \cdot l(s)=0$.

Hence it is enough to show the following lemma.

\section{Lemma 1.}

$$
\sum_{s=1}^{s=i}(-1)^{s}(s-1) ! d(s, i)=0 .
$$

Proof. Let us extend the definition of $d(s, i)$ for $s>i$ and $s \leq 0$, putting $d(s, i)=0$ in these cases. Notice that $d(s, i)=d(s-1, i-1)+s \cdot d(s, i-1)$ unless $s=i=1$. Indeed, the number $i$ can form a group itself, or it can belong to any of the groups formed by the other numbers. We have

$$
\begin{aligned}
\sum_{s=1}^{s=i}(-1)^{s}(s-1) ! d(s, i)= & \sum_{s=1}^{s=i}(-1)^{s}(s-1) ![d(s-1, i-1)+s \cdot d(s, i-1)] \\
= & \sum_{s=1}^{s=i}(-1)^{s}[(s-1) ! d(s-1, i-1)+s ! d(s, i-1) \\
& -d(0, i-1)+(-1)^{i} i ! d(i, i-1)=0 .
\end{aligned}
$$

Proof of the Claims. Claim 1 follows from the fact that $\bigcup B_{\alpha}$ is the transverse preimage of the diagonal $\Delta_{i}\left(R^{n+k}\right)$, and therefore the homology class realized by this union is zero.

Proof of Claim 3. Let us recall that in the partition $\alpha$ the groups are $I_{1}, \ldots, I_{s}$ and that $S$ was a set containing one element from each of these groups. For $1 \leq j \leq s$ let us denote by $\Delta_{I_{j}}\left(R^{n+k}\right)$ the subset of $\left(R^{n+k}\right)^{i}$ formed by those points $\left\{x_{1}, \ldots, x_{i}\right\}$ in which $x_{u}=x_{v}$ if $u \in I_{j}$ and $v \in I_{j}$. Similarly define the set $\Delta_{S}\left(R^{n+k}\right)=$ $\left\{x_{1}, \ldots, x_{i} \mid x_{u}=x_{v}\right.$ if $\left.u \in S, v \in S\right\}$. Clearly

$$
\Delta_{i}\left(R^{n+k}\right)=\bigcap_{j=1}^{s} \Delta_{I_{j}}\left(R^{n+k}\right) \cap \Delta_{S}\left(R^{n+k}\right) .
$$


Now we can make $\tilde{F}_{i}$ transverse to $\Delta_{i}\left(R^{n+k}\right)$ by making it transverse to each $\Delta_{I_{j}}\left(R^{n+k}\right)$ and $\Delta_{S}\left(R^{n+k}\right)$. Therefore

$$
F_{i}^{-1}\left(\Delta_{i}\left(R^{n+k}\right)\right)=\bigcap_{j=1}^{s} F_{i}^{-1}\left(\Delta_{I_{j}}\left(R^{n+k}\right)\right) \cap F_{i}^{-1}\left(\Delta_{S}\left(R^{n+k}\right)\right) .
$$

Intersecting both sides with $M_{\alpha}$, one gets the required equality.

Proof of Claim 2. Denote by $\xi$ the Whitney sum of $(i-1)$ copies of the tangent bundle of $M$, i.e. $\xi=(i-1) T M$, and let $\eta$ be the Whitney sum of $(i-1)$ copies of the tangent bundle of $R^{n+k}$, i.e. $\eta=(i-1) T R^{n+k}$. (Of course $\eta$ is just a trivial bundle, but we want to think of it in this way.) Now notice that the tubular neighbourhoods of $\Delta_{i}(M)$ and $\Delta_{i}\left(R^{n+k}\right)$ in $(M)^{i}$ and $\left(R^{n+k}\right)^{i}$ respectively can be identified with the bundles $\xi$ and $\eta$ respectively, and the map $\tilde{F}_{i}=f \times \cdots \times f$ : $(M)^{i} \rightarrow\left(R^{n+k}\right)^{i}$ can be identified with the map $G=d f \oplus d f \oplus \ldots d f .((i-1)$ summands $d f$. The map $G$ restricted to the zero section coincides with $f$.) Now we want to make $G$ transverse to the zero section of $\eta$ by a small perturbation. At a point $f(x)$ lying in the zero section of $\eta$ choose in each of the $(i-1)$ summands of $\eta$ isomorphic to $T R^{n+k}$ the complement of the image of $d f$, i.e the fiber $\nu_{f(x)}$ of the

normal bundle $\nu$ of $f$, and denote by $\theta_{f(x)}$ their direct sum, i.e $\theta_{f(x)}=(i-1) \nu_{f(x)}$. This linear space $\theta_{f(x)}$ "measures" the non-transversality of $G$ to the zero section at the point $f(x)$ in the sense that it is isomorphic to the quotient of the tangent bundle of the target manifold (= the total space of $\eta$ ) by the subspace generated by the image of the differential of $G$ and the tangent space of the zero section. Therefore if we choose a small perturbation $s$ of the zero section of the bundle $\theta=(i-1) \nu$ transverse to the zero section, then we can obtain from this easily a perturbation of $G$ transverse to the zero section of $\eta$. It is well known that the zero set $S$ of such a section $s$ realizes the homology class dual to the Euler class $\bar{e}^{i-1}$ in $H^{*}(M)$.

Now it remains to find the image of the homology class $[S] \in H_{*}(M)$ at the map induced by the "diagonal embedding" $j: M \rightarrow(M)^{i}$ defined by $j(x)=(x, x, \ldots, x)$.

Lemma 2. If $v \in H^{*}(M)$ is the cohomology class dual to the homology class realized by a submanifold $[V] \subset M$, then the cohomology class of $(M)^{i}$ dual to the homology class realized by $j(V)$ is the product $(v \times 1 \times \cdots \times 1) \cup \delta_{i}$. Recall that $\delta_{i}$ is the class dual to the diagonal $\Delta_{i}(M)$.

Proof. The manifold $j(V)$ is the (transverse) intersection of $\Delta_{i}(M)$ and the preimage of $V$ at the projection $(M)^{i} \rightarrow M$.

Thus the Main Lemma is proved, but in order to use it properly we need to find the diagonal cohomology class $\delta_{i}$. Let $\left\{c_{j}\right\}$ be a basis in $H^{*}(M)$ and let $\left\{c_{j}^{*}\right\}$ be the dual basis. Then $\left\langle c_{j} \cup c_{l}^{*},[M]\right\rangle=\delta_{j l}$, where $\delta_{j l}$ is the usual Kronecker delta.

Lemma 3. The diagonal class is

$$
\delta_{i}=\sum_{j_{1}, j_{2}, \ldots, j_{i}} c_{j_{1}} \times\left(c_{j_{1}}^{*} \cup c_{j_{2}}\right) \times\left(c_{j_{2}}^{*} \cup c_{j_{3}}\right) \times \cdots \times\left(c_{j_{i-1}}^{*} \cup c_{j_{i}}\right) \times c_{j_{i}}^{*} .
$$

where the classes $c_{j_{1}}, c_{j_{2}}, \ldots, c_{j_{i}}$ run over the basis of $H^{*}(M)$ independently.

Proof. This lemma can be found in [11] for $i=2$. The general case follows easily by the formula $\delta_{i}=\left(\delta_{2} \times 1 \times \cdots \times 1\right) \cup\left(1 \times \delta_{2} \times 1 \cdots \times 1\right) \cup \cdots \cup\left(1 \times \cdots \times 1 \times \delta_{2}\right)$. 


\section{§3. Proofs of the Theorems}

\subsection{The signature of the $i$-tuple manifold.}

Proof of Theorem 4. Since there is an orientation preserving $i$-fold covering $\tilde{\Delta}_{i}(f)$ $\rightarrow \Delta_{i}(f)$ we have $\sigma(\Delta)=\frac{1}{i !} \sigma\left(\tilde{\Delta}_{i}(f)\right)$, and so it is enough to find the signature of $\tilde{\Delta}_{i}(f)$. The inclusion $j: \tilde{\Delta}_{i}(f) \subset(M)^{i}$ has trivial normal bundle and realizes the homology class dual to the cohomology class

$$
b_{i}=(-1)^{i-1}(i-1) ! \cdot\left(\bar{e}^{i-1} \times 1 \times \cdots \times 1\right) \cup \delta_{i} .
$$

Let $\mathcal{D}$ denote the Poincaré duality operator of the manifold $(M)^{i}$. Therefore

$$
\begin{gathered}
\sigma\left(\tilde{\Delta}_{i}(f)\right)=\left\langle L\left(\tilde{\Delta}_{i}(f)\right),\left[\tilde{\Delta}_{i}(f)\right]\right\rangle=\left\langle j^{*}\left(L\left((M)^{i}\right)\right),\left[\tilde{\Delta}_{i}(f)\right]\right\rangle \\
=\left\langle L\left((M)^{i}\right), j_{*}\left[\tilde{\Delta}_{i}(f)\right]\right\rangle=\left\langle L\left((M)^{i}\right), \mathcal{D}\left(b_{i}\right)\right\rangle=\left\langle L\left((M)^{i}\right) \cup b_{i},\left[(M)^{i}\right]\right\rangle .
\end{gathered}
$$

Now using the equality $L\left((M)^{i}\right)=L(M) \times \cdots \times L(M)$, the fact that $b_{i}$ contains $\delta_{i}$ as a factor, and the symmetrizing property of the diagonal class, we obtain

$$
\begin{aligned}
& \left\langle L\left((M)^{i}\right) \cup b_{i},\left[(M)^{i}\right]\right\rangle \\
& \quad=(-1)^{i-1}(i-1) ! \cdot\left\langle\left(\left(\bar{e}^{i-1} \cup L^{i}(M)\right) \times 1 \times \cdots \times 1\right) \cup \delta_{i},\left[(M)^{i}\right]\right\rangle .
\end{aligned}
$$

Using the expression given for $\delta_{i}$ by the previous lemma, and the fact that the evaluation on the fundamental class $\left[(M)^{i}\right]$ can be done by coordinates, i.e. $\left\langle u_{1} \times u_{2} \times \cdots \times u_{i},\left[(M)^{i}\right]\right\rangle=\left\langle u_{1},[M]\right\rangle \cdot\left\langle u_{2},[M]\right\rangle \cdots\left\langle u_{i},[M]\right\rangle$, we see that

$$
\begin{aligned}
& \left\langle\left(\left(\bar{e}^{i-1} \cup L^{i}(M)\right) \times 1 \times \cdots \times 1\right) \cup \delta_{i},\left[(M)^{i}\right]\right\rangle \\
& =\sum_{j_{1}, j_{2}, \ldots, j_{i}}\left\langle\bar{e}^{i-1} \cup L^{i}(M) \cup c_{j_{1}},[M]\right\rangle \\
& \quad \cdot\left\langle c_{j_{1}}^{*} \cup c_{j_{2}},[M]\right\rangle \cdot\left\langle c_{j_{2}}^{*} \cup c_{j_{3}},[M]\right\rangle \ldots\left\langle c_{j_{i-1}}^{*} \cup c_{j_{i}},[M]\right\rangle\left\langle c_{j_{i}}^{*},[M]\right\rangle .
\end{aligned}
$$

All summands but one are zero in this sum. Indeed the last factor $\left\langle c_{j_{i}}^{*},[M]\right\rangle$ is not zero if and only if $c_{j_{i}}^{*}$ is the generator of $H^{n}(M)$, and then this factor is 1 . Then $c_{j_{i}}=1$. Therefore the previous factor is $\left\langle c_{j_{i-1}}^{*},[M]\right\rangle$. Again this is nonzero only if $c_{j_{i-1}}=1$. Finally we obtain that this sum is equal to $\left\langle\bar{e}^{i-1} \cup L^{i}(M),[M]\right\rangle$.

3.2 Proof of Theorem 2. Again we can suppose that $n$ is even. (Otherwise the manifolds $\Delta_{i}(f)$ have odd dimensions and so their Euler characteristics vanish.) Since $n+k \not \equiv 0 \bmod 4$, the manifolds $\Delta_{2 i}(f)$ are oriented manifolds of dimensions $\equiv 2 \bmod 4$ and therefore they have even Euler characteristics. The manifolds $\Delta_{2 i+1}(f)$ have dimensions divisible by 4 and so their Euler characteristics have the same parity as their signatures. By Theorem 4

$$
\sigma\left(\Delta_{2 i+1}(f)\right)=\frac{1}{2 i+1}\left\langle\bar{P}_{k / 2}^{i} L^{2 i+1}(M),[M]\right\rangle .
$$

The right hand side is a linear combination of the Pontrjagin numbers of $M$ with rational coefficients. These rational coefficients have odd denominators by a theorem of Atiyah and Hirzebruch saying that the coefficients of the $L$ class have this property, (see [2] or lemma 1.5.2 in [10]), and the Pontrjagin numbers of $M^{n}$ are even by Stong's theorem. Since the signature is an integer it must be an even number. 
3.3 Proof of Theorem 3. The total normal Pontrjagin class of $M^{n}$ is $\bar{P}(M)=1+$ $\bar{P}_{1}(M)$. The total normal Pontrjagin class of $\left(M^{n}\right)^{i}$ is $\bar{P}\left((M)^{i}\right)=\left(1+\bar{P}_{1}(M)\right) \times$ $\left(1+\bar{P}_{1}(M)\right) \times \cdots \times\left(1+\bar{P}_{1}(M)\right)$. Therefore $\bar{P}_{j}\left((M)^{i}\right)=\left(\begin{array}{l}i \\ j\end{array}\right) \cdot\left(\bar{P}_{1}(M) \times \cdots \times \bar{P}_{1}(M)\right)$. Multiplying this equality by $\delta_{i}$ and using the symmetrising property of the diagonal class, we get the following:

$$
\bar{P}_{j}\left((M)^{i}\right) \cup \delta_{i}=\left(\begin{array}{l}
i \\
j
\end{array}\right) \cdot\left(\left(\bar{P}_{1}(M)\right)^{j} \times 1 \times \cdots \times 1\right) \cup \delta_{i} .
$$

If $J=\left(j_{1}, \ldots, j_{r}\right)$, then similarly

$$
\bar{P}_{J}\left((M)^{i}\right) \cup \delta_{i}=\left(\begin{array}{c}
i \\
J
\end{array}\right) \cdot\left(\bar{P}_{1}(M)^{\left(j_{1}+\cdots+j_{r}\right)} \times 1 \times \cdots \times 1\right) \cup \delta_{i} .
$$

Now the proof is very similar to that of Theorem 4. Namely:

Let $j: \tilde{\Delta}_{i}(f) \subset(M)^{i}$ be the inclusion. Then

$$
\begin{gathered}
\left\langle\bar{P}_{J}\left(\tilde{\Delta}_{i}(f)\right),\left[\tilde{\Delta}_{i}(f)\right]\right\rangle=\left\langle j^{*}\left(\bar{P}_{J}\left((M)^{i}\right),\left[\tilde{\Delta}_{i}(f)\right]\right\rangle\right. \\
=\left\langle\bar{P}_{J}\left((M)^{i}\right) \cup b_{i},\left[(M)^{i}\right]\right\rangle=\left\langle\bar{P}_{J}\left((M)^{i}\right) \cup\left(\bar{e}^{i-1} \times 1 \times \cdots \times 1\right) \cup \delta_{i},\left[(M)^{i}\right]\right\rangle .
\end{gathered}
$$

Substituting for $\delta_{i}$ in $(*)$ its value given in Lemma 3 and using the fact that, on evaluating by coordinates in the resulting sum, there is (at most) one nonzero summand (see the argument in the proof of Theorem 2), one gets that the last expression is equal to $\left(\begin{array}{l}i \\ J\end{array}\right)\left\langle\bar{P}_{1}(M)^{j_{1}+j_{2}+\cdots+j_{r}} \cup \bar{e}^{i-1},[M]\right\rangle$ This implies the first equality in Theorem 3 if we recall that $\bar{e}^{2}=\bar{P}_{1}(M)$. The second one follows by Herbert's formula [9] (see also Remark 5)

The proof of Theorem 5 , is completely analogous.

\section{REFERENCES}

[1] Akhmetiev, P. - Rimányi, R. - Szücs, A. A generalization of Banchoff's theorem, To appear in Proc. Amer. Math. Soc.

[2] Atiyah, M. - Hirzebruch, F. Cohomologie - Operationen und characteristische Klassen, Math. Zeitschrift., 77 (1961), 149 - 187. MR 27:6285

[3] Banchoff, T. Triple points and surgery of immersed surfaces, Proc. Amer. Math. Soc. 46 (1974) 407 - 413. MR 51:14066

[4] Eccles, P.J. Multiple points of codimension one immersions of oriented manifolds, Math. Proc. Cambridge Phil. Soc. 87 (1980) 213 - 220. MR 81j:55014

[5] Eccles, P.J. Codimension one immersions and the Kervaire invariant one problem, Math. Proc. Cambridge Philos. Soc. 90 (1981) 483 - 493. MR 83c:57015

[6] Eccles, P. J. Double point manifolds of immersions of spheres in Euclidean space, in Prospects of Topology (editor: Frank Quinn) Annals of Math. Studues, 138 (1995) 125 - 137. MR 96m:57044

[7] Lannes, J. Sur les immersions de Boy, In Algebraic Topology, AArhus 1982, Lecture Notes in Math. vol. 1051 (Springer - Verlag, 1984) pp. 263 - 270. MR 86d:57015

[8] Lashof, R. and Smale, S. Self intersections of immersed manifolds, Journal of Math. and Mech. 8 (1959), 143 - 157. MR 21:332

[9] Herbert, R. J. Multiple points of immersed manifolds, Memoirs AMS Nov. 1981, vol.34. No. 250. MR 84c:57022

[10] Hirzebruch, F. Topological methods in Algebraic Geometry, Springer-Verlag New York 1966. MR 34:2573

[11] Milnor, J.W. and Stasheff J.D. Characteristic classes, Ann. of Math. Studies 76, Princeton (1974). MR 55:13428

[12] Stong, R. Manifolds which immerse in small codimension, Illinois Journ. of Math. vol 27, Number 2, Summer 1983. MR 85b:57037 
[13] Ronga, F. On multiple points of smooth immersions, Comment. Math. Helv. 55 (1980) (521527). MR 82d:57018

[14] Szücs, A. Double point surfaces of smooth immersions $M^{n} \rightarrow R^{2 n-2}$, Math. Proc. Camb. Phil. Soc. (1993), 113, 601 - 613. MR 94e:57047

[15] Szücs, A. Cobordism of maps with simplest singularities, Topology Symposium, Siegen 1979. Lecture Notes in Math. vol 788. (Springer-Verlag 1980), 223-244. MR 82b:57025

[16] Szücs, A. Cobordism groups of immersions of oriented manifolds, Acta Math. Hungarica 64 (2) (1994) 191-230. MR 95e:57050

[17] Wells, R. Double covers and metastable immersions of spheres, Canad. Journ. of Math. 26 (1974) 145 - 176. MR 49:3981

Department of Analysis, Elte, Muzeum krt. 6-8, Budapest, 1088 Hungary

E-mail address: szucsandras@ludens.elte.hu 